\title{
Estimation of Neutrino Masses Without Using Seesaw Mechanism
}

\author{
Teruo Kurai \\ Fuchuu-shi, Tokyo, Japan \\ Email address: \\ kurai.teruo@topaz.plala.or.jp \\ To cite this article: \\ Teruo Kurai. Estimation of Neutrino Masses Without Using Seesaw Mechanism. International Journal of High Energy Physics. \\ Vol. 6, No. 2, 2019, pp. 54-60. doi: 10.11648/j.ijhep.20190602.14
}

Received: December 8, 2019; Accepted: December 16, 2019; Published: December 24, 2019

\begin{abstract}
We propose the Bethe-Salpeter-like amplitude of spin operator in spin space and consider that the vibration of this spin operator amplitude causes the vibration in azimuthal angle space, which causes the anomalous magnetic moment of leptons and generates masses of flavor state neutrino. Under this consideration, we can estimate neutrino masses using anomalous magnetic moment of leptons instead of using conventional seesaw mechanism. Electron anomalous magnetic moment and muon anomalous magnetic moment have been measured precisely so that we can estimate the masses of electron and muon neutrino systemically in our consideration. For tau neutrino mass case, we cannot estimate it in our consideration because tauon anomalous magnetic moment has not been measured. Instead, we use the squared mass splitting data to estimate tau neutrino mass in this paper. These are not mass eigenstates masses but flavor states masses, however, the sum of these masses, which should be equal to the sum of mass eigen states masses, is consistent to the current upper and lower bound of the sum of neutrino masses for both cases of normal hierarchy and inverted hierarchy.
\end{abstract}

Keywords: Beth-Salpeter-like Amplitude, Spin Operator, Neutrino Mass

\section{Introduction}

The oscillation experiments in the late 1990s, such as Super- Kaminokande [1]. And recent experiments, such as SNO [2], KamLAND [3], and others [4-6], have established the existence of massive neutrino. Then, it becomes the fundamental target to find the absolute values of neutrino mass $\left.v_{i}(i=1,2,3)\right)$. Lourero A. et al. [7] investigate the impact of prior models on the upper bound of the sum of neutrino masses, $\sum m_{v}$ and obtain a consistent upper bound of $\sum m_{v} \lesssim 0.26 \mathrm{eV}(95 \% \mathrm{CI})$ within the $\Lambda \mathrm{CDM}$ concordance model. Another model dependent method is provided by the search for neutrinoless double $\beta$ - decay, $0_{v} \beta \beta$, a process forbidden in the Standard Model (SM) due to lepton number violation. It gives access to the effective Majorana neutrino masses $[8,9]$. A model-independent, direct method to measure the neutrino mass is provided by kinematics studies of $\beta$ - decay of tritium $(H)$ and electron capture on holmium (Ho) [10-13]. In this type of experiment, KATRIN [14] recently reports the upper bound of $m_{v}<1.1 \mathrm{eV}$. In theory side, the smallness of neutrino masses is explained by seesaw mechanism [15-19]. In this scheme, Morisi S. et al. [20, 21] shows to arise the neutrino masses in the supersymmetric model. However, there has been no article (at least in our knowledge) to specify the absolute neutrino masses. In this paper, we show the estimated value of neutrino masses using by rather different consideration.

\section{Formalism}

Our approach to estimate neutrino masses is based on the consideration that neutrino spins generate those masses. To work along this consideration, we first propose that spin is generated by the vibration in spin space and that spin space is not real physical space but is related to the vibration of azimuthal (rotational) angle $\varphi$ which is real physical space. We consider that this vibrational mode generates anomalous magnetic moments and can be used to explain the spin magnetic moment which is related to mass of neutrino.

To be specific, we speculate the spin operator resemble to the hadronic operator proposed by Suura $[22,23]$ in spin space as follows.

We define the Bethe-Salpeter-like amplitude as

$$
\chi_{s}=<0|s(1,2)| p s>
$$


where $\mid 0>$ and $\mid \mathrm{ps}>$ denote the vacuum and physical states in spin space, respectively, and the gauge invariant bi-local operator in the non-Abelian gauge field as

$$
\mathrm{s}_{\alpha \beta}(1,2)=T_{r}^{c} e_{s \beta}^{\dagger}(2) P \exp \left(i g_{s} \int_{1}^{2} \overrightarrow{d s} \vec{A}^{a}(\vec{s}) \frac{\lambda_{a}}{2}\right) \mathrm{e}_{s \alpha}
$$

Here $e_{s}$ and $\overline{e_{s}}$ denote the spin particle and anti-spin particle and $\alpha, \beta$ denote the Dirac indices. P denotes the path ordering and the $\frac{\lambda_{a}}{2}$ components are generators of adjoint representation of the SU $(\mathrm{N})$ color gauge group. The trace is calculated for the color spin a.

The defined operator of Eq. (2) is gauge invariant but path-dependent. We adopt the straight line for the path. The reason of this choice is given in Ref. [24]. Then, the equation of motion of Eq. (2) becomes exactly same form as that of hadronic operator obtained in Ref. [24]. Therefore, we can obtain the same wave functions in the relative coordinate system except the variable is not $\mathrm{r}$ but $\mathrm{s}$ defined as $\mathrm{s}=$ $\sqrt{s_{x}^{2}+s_{y}^{2}+s_{z}^{2}}$.

In Ref. [24], the explicit form of equations are given as

$$
\begin{gathered}
\chi_{s 0}(\mathrm{~s})=\chi_{s 1}(s)=0 \\
0=\frac{\partial^{2} \chi_{s 2}}{\partial s^{2}}+\frac{1}{s} \frac{\partial \chi_{s 2}}{\partial s}+\left(-\frac{g_{s}^{2} L_{1}}{2}+\frac{1}{4}\left(P_{0}-\frac{\frac{g_{s}^{2} L_{1}}{2}}{P_{0}}\right)^{2}\right) \chi_{s 2}- \\
\frac{1}{4}\left(\frac{g_{s}^{2} L_{1}}{2}\right)^{2} s^{2} \chi_{s 2} \\
0=\frac{\partial^{2} \chi_{s 3}}{\partial s^{2}}+\frac{1}{s} \frac{\partial \chi_{s 3}}{\partial s}-\frac{1}{s^{2}} \chi_{s 3}+\frac{1}{4}\left(P_{0}-\frac{\frac{g_{s}^{2} L_{1}}{2}}{P_{0}}\right)^{2} \chi_{s 3}- \\
\frac{1}{4}\left(\frac{g_{s}^{2} L_{1}}{2}\right)^{2} s^{2} \chi_{s 3}
\end{gathered}
$$

Here we set $\delta(0)=1$ because we use equal time commutation relation.

The solutions of Eq. (4) and Eq. (5) are obtained for $\mu=+\frac{1}{2}$ case as

$$
\begin{gathered}
\chi_{s 2}(\mathrm{~s})=\mathrm{const} \exp \left(-\frac{g_{s}^{2} L_{1}}{8} s^{2}\right) F\left(\frac{1}{2}-\kappa, 1 ; \frac{g_{s}^{2} L_{1}}{4} s^{2}\right) \\
\chi_{s 3}(s)=\text { const } \operatorname{sexp}\left(-\frac{g_{S}^{2} L_{1}}{8} s^{2}\right) F\left(1-\kappa, 2 ; \frac{g_{S}^{2} L_{1}}{4} s^{2}\right)
\end{gathered}
$$

where $\quad \kappa=-\frac{1}{2}+\frac{1}{4 g_{S}^{2} L_{1}}\left(P_{0}-\frac{\frac{g_{S}^{2} L_{1}}{2}}{P_{0}}\right)^{2}$ for Eq. (6) and $\kappa=\frac{1}{4 g_{S}^{2} L_{1}}\left(P_{0}-\frac{\frac{g_{S}^{2} L_{1}}{2}}{P_{0}}\right)^{2}$ for Eq. (7)

$$
\begin{aligned}
\left(P_{0}\right)^{2}=\frac{g_{s}^{2} L_{1}}{2}(1 & +4(n+1)) \\
& +\sqrt{\left(\frac{g_{s}^{2} L_{1}}{2}(1+4(n+1))\right)^{2}-\left(\frac{g_{s}^{2} L_{1}}{2}\right)^{2}}
\end{aligned}
$$

( $\mathrm{n}$ is 0 or positive integer)
$\mathrm{F}(\alpha, \gamma ; z)$ is the Kummer's confluent hypergeometric series defined by [25]

$$
\mathrm{F}(\alpha, \gamma ; z)=\sum_{n=0}^{\infty} \frac{\alpha(\alpha+1) \cdot(\alpha+n-1)}{\gamma(\gamma+1) \cdot(\gamma+n-1)} \frac{z^{n}}{n !}
$$

Important point that the wave functions of Eq. (6) and Eq. (7) include the harmonic oscillator type wave functions as

$$
\begin{aligned}
& \chi_{s_{2}}(s)=\text { const } \exp \left(-\frac{g_{S}^{2} L_{1}}{8} s^{2}\right) \\
& \chi_{s_{3}}(s)=\text { const } \operatorname{sexp}\left(-\frac{g_{S}^{2} L_{1}}{8} s^{2}\right)
\end{aligned}
$$

Note that the solutions Eq. (9) and Eq. (10) come from the $\kappa=\frac{1}{2}$ case of Eq. (6) and the $\kappa=1$ case of Eq. (7), respectively.

Reminding that $\chi_{s}(s)$ is decomposed as

$$
\begin{aligned}
\chi_{s}(s)= & 1 \chi_{s 0}(s)+(-i \vec{\alpha} \cdot \hat{s}) \chi_{s 1}(s)+\beta \chi_{s 2}(s)+ \\
& \beta(i \alpha \cdot \hat{s}) \chi_{s 3}(s)
\end{aligned}
$$

We can notice that Eq. (9) and Eq. (10) are the wave functions of $\beta$ component and that of $\beta(i \vec{\alpha} \cdot \hat{s})$ component, respectively.

Here, $\alpha^{k}=\gamma^{0} \gamma^{k}, \beta=\mathrm{i} \gamma^{0}$ and $\gamma$ matrices are defined as

$$
\gamma^{0}=(-i)\left[\begin{array}{cc}
0 & \sigma_{0} \\
\sigma_{0} & 0
\end{array}\right], \gamma^{k}=(-i)\left[\begin{array}{cc}
0 & \sigma_{k} \\
\sigma_{k} & 0
\end{array}\right](\mathrm{k}=1,2,3)
$$

employed by Weinberg [26]. Here, $\sigma_{0}$ is unit matrix of $2 \times 2$ matrix and $\sigma_{k}$ is the $2 \times 2$ Pauli-matrices.

Although Eq. (9) and Eq. (10) are one of the solutions of Eq. (4) and Eq. (5), respectively, we can also consider that these are the solutions of Weber equation of which the standard form is defined as [25]

$$
\frac{\partial^{2}}{\partial z^{2}} D_{n}(z)+\left(\left(n+\frac{1}{2}\right)-\frac{z^{2}}{4}\right) D_{n}(z)=0
$$

where $\mathrm{n}$ is 0 or positive integer

The solution of Eq. (12) is expressed as

$$
D_{n}(z)=(-1)^{n} \exp \left(\frac{z^{2}}{4}\right) \frac{d^{n}}{d z^{n}} \exp \left(-\frac{z^{2}}{2}\right)
$$

From Eq. (13), we can notice that Eq. (9) and Eq. (10) are also the solutions of Eq. (12) for $n=0$ and $n=1$ case, respectively, under $\mathrm{z}=\sqrt{\frac{g_{S}^{2} L_{1}}{2}} S$.

Taking the variable as $\mathrm{z}=\sqrt{2}\left(\frac{1}{\left(\hbar^{2} k\right)(2 I)}\right)^{\frac{1}{4}} s$, from Eq.(12), we can obtain the Shrödinger equation form as

$$
-\hbar^{2} k \frac{\partial^{2} W(s)}{\partial s^{2}}+\frac{s^{2}}{2 I} W(s)=2 \sqrt{\frac{k}{2 I}} \hbar\left(n+\frac{1}{2}\right) W(s)
$$

The important point is that the $\mathrm{n}=1$ vibrational mode comes from the solution of $\beta(i \vec{\alpha} \cdot \hat{s})$ component, that is, $\mathrm{i} \gamma^{k}$ matrix solution of which matrix elements are composed by Pauli-matrices. Thus, it is reasonable to consider that the $n=1$ 
vibrational mode represents the vibration in spin space. Also, Pauli-matrices in $\gamma^{k}$ matrix appear in off-diagonal block matrices. This indicates that the proposed spin space is not real because Dirac showed that Pauli-matrices in diagonal block matrices represent the actual spin angular moment [27].

Here, we consider that spin $\mathrm{s}$ and azimuthal (rotational) angle $\phi$ are canonical conjugate each other. Thus, the commutation relation between $\phi$ and $\mathrm{s}$ becomes as

$$
[\phi, s]=i \hbar
$$

This means that the relation between $\phi$ and $\mathrm{s}$ is expressed as

$$
\begin{aligned}
& \mathrm{s} \leftrightarrow-\mathrm{i} \hbar \frac{\partial}{\partial \phi} \\
& \phi \leftrightarrow \mathrm{i} \hbar \frac{\partial}{\partial s}
\end{aligned}
$$

Using Eq. (16) and Eq. (17), Eq. (14) becomes

$$
-\frac{\hbar^{2}}{2 I} \frac{\partial^{2} \psi(\phi)}{\partial \phi^{2}}+k \phi^{2} \psi(\phi)=2 \sqrt{\frac{k}{2 I}} \hbar\left(n+\frac{1}{2}\right) \psi(\phi)
$$

Eq. (18) is the Shrödinger equation of harmonic oscillator with momentum inertia I and eigen value (vibrational energy) of $E_{\phi n}=2 \sqrt{\frac{k}{2 I}} \hbar\left(n+\frac{1}{2}\right)$.

Note that this vibrational energy is the same vibrational energy in spin space as shown in Eq. (14). This means that the vibrational mode in spin space corresponds to that of azimuthal angle $\phi$ space.

\section{Estimation}

In section 2, we show that a vibrational mode in spin space generates that of azimuthal angle $\phi$ space using by quantum theory consideration. To estimate neutrino masses, however, we describe the phenomena by classical physics. Classically, electron spin, muon spin and tauon spin are described by the result of the spinning charged particles. To be specific, we consider electron case.

We consider electron as a spinning rigid body sphere with the charge $e^{-}$evenly distributed in the surface. In electro-magnetism, the magnetic moment is defined as

$$
\vec{\mu}=\iiint d V \vec{r} \times \vec{\jmath}
$$

Considering the angular velocity (rotational velocity) $\dot{\phi}=\omega$ (dot denotes time derivative) and the radius $a_{0}$, the electron magnetic moment is calculated as

$$
\begin{array}{r}
\mu_{e z}= \\
\int_{0}^{a_{0}} r^{2} d r \delta\left(r-a_{0}\right) \int_{0}^{\pi} \sin ^{2} \theta d \theta \int_{0}^{2 \pi} d \phi r^{2} \sin ^{2} \theta \rho(e) \omega= \\
\text { (20) } \omega a_{0}^{2}
\end{array}
$$

where $\rho(e)$ is the electron density of $\frac{(-e)}{4 \pi a_{0}^{2}}$ and $\mathrm{z}$ axis is taken along the rotation axis.

From now on, we consider $a_{0} \omega=\bar{\omega}$ as the rotational (angular) velocity.
The electron spin magnetic moment is written as

$$
\begin{gathered}
\overrightarrow{\mu_{e}}=\frac{(-e)}{2 M_{e}} \vec{s} \hbar \\
\mu_{e z}=\frac{(-e)}{2 M_{e}} \frac{1}{2} \hbar\left(s_{z}=\frac{1}{2}\right)
\end{gathered}
$$

where $M_{e}$ is electron mass.

From Eq. (20) and Eq. (22), and using the relation that

$$
M_{e} c^{2}=\frac{2 \alpha \hbar c}{a_{0}}
$$

we obtain

$$
\begin{gathered}
\frac{2}{3} \mathrm{e} \bar{\omega} a_{0}=\frac{e}{2 M_{e}} \frac{1}{2} \hbar \\
\bar{\omega}=\frac{3}{16} \frac{c}{\alpha}
\end{gathered}
$$

where

$$
\alpha=\frac{e^{2}}{4 \pi \varepsilon_{0} \hbar c}=\frac{1}{137.04}, \quad c: \text { light velocity }
$$

Note that the factor 2 of Eq. (23) comes from the consideration that both the electric energy $U_{e}$ and the magnetic energy $U_{m}$ contribute to electron mass equally, that is, one half of electron mass is generated by the electric energy and the other half is given by the magnetic energy.

The interaction energy is defined as

$$
\begin{gathered}
U_{\text {int }}=-\vec{\mu} \cdot \vec{B}=-\mu_{z} B_{z} \\
\vec{\mu}=-\frac{\partial U_{i n t}}{\partial \vec{B}}, \mu_{z}=-\frac{\partial U_{i n t}}{\partial B_{z}}
\end{gathered}
$$

From Eq. (20), Eq. (26) and Eq. (27), we notice that $B_{z}$ is obtained as

$$
B_{z}=-\mu_{0} \mu_{z}=\mu_{0} \frac{2}{3} e \bar{\omega} a_{0}
$$

where $\mu_{0}$ is permeability of free space.

Thus, the interaction energy becomes

$$
U_{i n t}=\mu_{0}\left(\frac{2}{3} e \bar{\omega}\right)^{2} a_{0}^{2}
$$

Because the dimension of $U_{\text {int }}$ is $\mathrm{eV} \cdot L^{3}$, the magnetic energy is obtained as

$$
U_{m}=U_{i n t} / \frac{4 \pi}{3} a_{0}^{3}=\mu_{0}\left(\frac{2}{3} e \bar{\omega}\right)^{2} \frac{3}{4 \pi} \frac{1}{a_{0}}
$$

Reminding that Eq. (18) is Schrödinger equation of harmonic oscillator, Eq. (18) can be expressed in classical mechanics as

$$
\mathrm{I} \ddot{\phi}+k \phi=0
$$

The solution of Eq. (31) becomes

$$
\phi=A \sin \left(\sqrt{\frac{k}{I}} t\right)
$$


Thus, the rotational velocity becomes

$$
\dot{\bar{\phi}}=a_{0} \dot{\phi}=a_{0} A \sqrt{\frac{k}{I}} \cos \left(\sqrt{\frac{k}{I}} t\right)
$$

This infinitesimal vibration causes the vibrational change of the magnetic field (B-field) in the electron and this B-field vibration is smoothly continued by not only the spin space vibration but the electromagnetic induction. Thus, the vibration of B-field can be also expressed as

$$
\Delta B_{z}=\Delta B_{0} \cos \left(\sqrt{\frac{k}{I}} t\right)
$$

From Eq. (33), we can express $\Delta B_{z}$ as

$$
\Delta B_{z}=\text { const }\left|\bar{A} \sqrt{\frac{k}{I}}\right| \cos \left(\sqrt{\frac{k}{I}} t\right)
$$

where $\bar{A}=a_{0} A$

Here, we consider that the vibration of azimuthal angle $\phi$ caused by the vibrational mode in spin space generates the anomalous magnetic moments mentioned in section 2 .

Eq. (25) shows that the obtained angular velocity is unphysical because it is larger than light velocity. Thus, to deal with the magnetic moment correctly, we have to rewrite the corresponding B-field as follows.

Because we can rewrite rotational velocity as $\alpha \bar{\omega}=\frac{3}{16} c$, the corresponding B-field can be rewritten as

$$
B_{z}=\mu_{0} \frac{2}{3} e(\alpha \bar{\omega})\left(\frac{1}{\alpha} a_{0}\right)
$$

Using our consideration as mentioned before, the anomalous magnetic moment can be given as follows because $\mathrm{B}$-field is related to the magnetic moment.

$$
\left(\frac{\Delta B_{Z}}{B_{Z}}\right)^{2}=\frac{\left(\mu_{0} \frac{2}{3} e\right)^{2}\left(\bar{A} \sqrt{\frac{k}{I}}\right)^{2} \cos ^{2}\left(\sqrt{\frac{k}{I}} t\right)\left(\frac{1}{\alpha} a_{0}\right)^{2}}{\left(\mu_{0} \frac{2}{3} e\right)^{2}(\alpha \bar{\omega})^{2}\left(\frac{1}{\alpha} a_{0}\right)^{2}}=\frac{\frac{1}{2}\left(\frac{1}{\alpha} \bar{A} \sqrt{\frac{k}{I}}\right)^{2}}{\bar{\omega}^{2}}=a_{e}^{2}
$$

where $a_{e}$ is the electron anomalous magnetic moment, and anomalous magnetic moment is defined as $\frac{g-2}{2}=a, g$ is factor of magnetic moment, the factor of $\frac{1}{2}$ in the second line comes from the mean value by time for cosine term.

We define the mean value by time as

$$
\begin{gathered}
<V>_{T}=\lim _{T \rightarrow \infty} \frac{1}{T} \int_{0}^{T} d t V \\
<\cos ^{2}\left(\sqrt{\frac{k}{I}} t\right)>_{T}=\lim _{T \rightarrow \infty} \frac{1}{T} \int_{0}^{T} d t \cos ^{2}\left(\sqrt{\frac{k}{I}} t\right) \\
=\lim _{T \rightarrow \infty} \frac{1}{T}\left(\frac{T}{2}-\frac{1}{4} \sqrt{\frac{I}{k}} \sin \left(2 \sqrt{\frac{k}{I}} T\right)\right)=\frac{1}{2}
\end{gathered}
$$

$$
\frac{1}{2}\left(\frac{1}{\alpha} \bar{A} \sqrt{\frac{k}{I}}\right)^{2}=\frac{a_{e}^{2} \frac{1}{2} M_{e} c^{2}}{\frac{3}{4 \pi} \mu_{0}\left(\frac{2}{3} e\right)^{2}} a_{0}
$$

Reminding the magnetic energy is expressed by Eq. (30), we obtain from Eq. (37)

where $<U_{m}>_{T}=\frac{1}{2} M_{2} c^{2}$

Note that precisely $\left\langle U_{m \text { total }}\right\rangle_{T}=\frac{1}{2} M_{e} c^{2}$ is correct, but $a_{e}^{2}$ is sufficiently small so that we adopt $\left\langle U_{m}>_{T}=\frac{1}{2} M_{2} c^{2}\right.$, where $<U_{m \text { total }}>_{T}=\mu_{0}\left(\frac{2}{3} e\right)^{2}\left((\alpha \bar{\omega})^{2}+\frac{1}{2}\left(\bar{A} \sqrt{\frac{k}{I}}\right)^{2}\right) \frac{1}{\frac{1}{\alpha} a_{0}}$.

Reminding that $\bar{A}=a_{0} A$ and $\mathrm{A}$ is order 1, and using Eq. (23) and Eq. (38), we obtain

$$
\mathrm{k}=\frac{6}{10} a_{e}^{2} M_{e} c^{2} \alpha^{2}
$$

For neutrino masses, we know that mass eigen states are different from flavor states such as electron, muon and tau neutrino. However, we consider the next estimated mass as the electron neutrino mass because of our consideration that the cause of its mass is the vibration in spin space.

For any neutrino, it has no electric charge so that we consider that B-field generated by the vibration in $\phi$ space causes the spin magnetic moment. To satisfy this consideration, we invoke the hypothetical electric $e^{+}$and $e^{-}$ charge distributed evenly on the surface of the neutrino, i.e, double layer surface, and assume that these two layers are rotating to opposite direction each other and the direction is oscillating. To keep the direction of spin magnetic moment one direction, neutrino itself is rolling up and down.

Reminding the relation equation Eq. (24), and using $\overline{\bar{\omega}}=a_{0}^{v e} \omega=\frac{1}{2}\left|\overline{\bar{A}} \sqrt{\frac{k}{I_{v e}}}\right|$, where $\overline{\bar{A}}=a_{0}^{v e} A, \mathrm{k}$ is given by Eq. (39),

$$
\frac{2}{3} \mathrm{e} \overline{\bar{\omega}} a_{0}^{v e}=\frac{e}{2 M_{v e}} \frac{1}{2} \hbar
$$

Here, we consider that the difference of angular velocity between $e^{+}$and $e^{-}$is $\overline{\bar{\omega}}$.

Then we obtain

$$
M_{v e} c^{2}=\frac{3}{8} \frac{\hbar c^{2}}{\overline{\bar{\omega}} a_{0}^{v e}}
$$

Because we consider the electron neutrino as the spinning hypothetical charged particle with spin magnetic moment of which rotational direction is oscillating, the electron neutrino mass becomes the sum of the vibrational energy and the magnetic energy.

In section 2 , we derive that the $\mathrm{n}=1$ vibrational mode is the actual vibrational mode in spin space and is related to the vibrational mode in $\phi$ space.

Therefore, the vibrational energy is $E_{v}=\hbar \sqrt{\frac{k}{2 I_{v e}}} 3$.

Then,

$$
\frac{1}{2} M_{v e} c^{2}=\hbar \sqrt{\frac{k}{2 I_{v e}}} 3
$$


From Eq. (42), we obtain

$$
a_{0}^{v e}=2 \sqrt{k} \frac{3 \hbar c}{\left(M_{v e} c^{2}\right)^{\frac{3}{2}}} \sqrt{\frac{5}{4}}
$$

Here, we use the momentum inertia as $I_{v e}=\frac{2}{5} M_{v e}\left(a_{0}^{v e}\right)^{2}$ for the rigid body sphere case.

Using Eq. (30), the contribution of magnetic energy to mass is expressed as

$$
\frac{1}{2} M_{v e} c^{2}=\mu_{o}\left(\frac{2}{3} e\right)^{2} \frac{3}{4 \pi} \frac{\overline{\bar{\omega}}^{2}}{a_{0}^{v e}}
$$

From Eq. (41) and Eq. (44), we obtain

$$
\overline{\bar{\omega}}=\left(\frac{1}{2}\right)^{\frac{1}{3}}\left(\frac{9}{32 \alpha}\right)^{\frac{1}{3}} \mathrm{c}
$$

Using Eq. (41), Eq. (43) and Eq. (45), we obtain the mass of electron neutrino as

$$
M_{v e} c^{2}=16 * 3^{\frac{4}{3}} * k * \frac{5}{4} *\left(\frac{1}{\alpha}\right)^{\frac{2}{3}}=4 * 3 * 3^{\frac{4}{3}} * a_{e}^{2} M_{e} c^{2} * \alpha^{\frac{4}{3}}
$$

For the last line, we use Eq. (39) for k.

Note that Eq. (45) shows this rotational velocity is also unphysical, however, the result, Eq. (45), is physical. The reason is given in Appendix.

To obtain the mass of mu-neutrino, we consider as follows.

Mu-neutrino has the same spin $\frac{1}{2}$ as electron neutrino and it has also no electric charge. Thus, it is reasonable to consider that the spin magnetic moment is generated by the vibration with same potential energy as electron in spin space, that is, $\mathrm{k}$ is same. The mass difference comes from the difference of their radius $a_{0}^{v e}, a_{0}^{v \mu}$. However, we cannot specify their radius. Then, we assume that $k_{v \mu}$ is infinitesimally different from $k_{v e}$, which is not actual cause of the difference of their radius, under the condition that $\overline{\overline{\omega_{v \mu}}}=\overline{\overline{\omega_{v e}}}$. Also, we assume that the infinitesimal difference of their $\mathrm{k}$ comes from the difference of their anomalous magnetic moment. Then, $k_{v \mu}$ is expressed as

$$
k_{v \mu}=\frac{6}{10} a_{\mu}^{2} M_{e} c^{2} \alpha^{2}
$$

where $a_{\mu}$ is muon anomalous magnetic moment.

Thus, the mass of mu-neutrino is obtained as

$$
M_{\nu \mu} c^{2}=4 * 3 * 3^{\frac{4}{3}} * a_{\mu}^{2} M_{e} c^{2} \alpha^{\frac{4}{3}}
$$

In the case of tau neutrino mass, we cannot use previous argument because there has been no experimental data of anomalous magnetic moment of tauon. Thus, we use the square mass difference between mass eigen states $v_{1}$ and $v_{3}$, Then, the mass of tau neutrino is given as

$$
M_{v \tau} c^{2}=\sqrt{\left(M_{v e} c^{2}\right)^{2}+m_{31}^{2}}
$$

\section{Results}

Table 1. Neutrino masses.

\begin{tabular}{lll}
\hline neutrino & $\begin{array}{l}\text { Mass (eV) anomalous } \\
\text { magnetic moment base }\end{array}$ & $\begin{array}{l}\text { Mass squared mass difference } \\
\text { base }\end{array}$ \\
\hline electron & $5.050 \times 10^{-2}$ & \\
mu & $5.105 \times 10^{-2}$ & $5.124 \times 10^{-2}$ \\
& & $\begin{array}{l}7.095 \times 10^{-2}(\mathrm{NH}) 0.0813 \times \\
10^{-2}(\mathrm{IH})\end{array}$ \\
\hline
\end{tabular}

Table 1 shows the list of neutrino masses estimated by Eq. (46), Eq. (48) and Eq. (49).

\begin{tabular}{|c|c|c|c|}
\hline & $\begin{array}{l}\text { Anomalous } \\
\text { magnetic moment }\end{array}$ & & $\begin{array}{l}\text { Squared mass [30] Difference } \\
\left(e V^{2}\right)\end{array}$ \\
\hline$a_{e}$ & $0.001159652[28]$ & $\Delta m_{21}^{2}$ & $7.49_{-0.17}^{+0.19} \times 10^{-5}$ \\
\hline$a_{\mu}$ & 0.001165920 [29] & $\left|\Delta m_{31}^{2}\right|$ & $2.484_{-0.048}^{+0.045} \times 10^{-3}$ \\
\hline
\end{tabular}

Here, $\mathrm{NH}$ and $\mathrm{IH}$ denote normal hierarchy and inverted hierarchy, respectively.

For the squared mass difference based mass of mu-neutrino, we use the same form as Eq. (49) adopting $m_{21}^{2}$ instead of $m_{31}^{2}$.

Table 2. Numerical value used for estimation.

Table 2. shows the numerical values used for our estimation. We also use the numerical value of $0.511(\mathrm{MeV})$ for electron mass and $\alpha=\frac{1}{137.04}$. Note that the squared mass difference of $\Delta m_{31}^{2}$ is positive value for $\mathrm{NH}$ case and is negative value for IH case.

To check the consistency of our results, we can compare the sum of three flavor neutrino masses to the sum of three mass eigen states masses because total mass should be equal.

Our results indicate that

$\sum m_{v}=0.1725 \mathrm{eV}(\mathrm{NH})$ and $\sum m_{v}=0.1024 \mathrm{eV}(\mathrm{IH})$. For the comparison, the latest article [7] shows that $\sum m_{v}<$ $0.264 \mathrm{eV}[95 \% \mathrm{CI}]$ as mentioned in the introduction. Also, there have been the reports which show a lower bound for the sum of neutrino masses [31-34]. Their results are as follows: $\sum m_{v}>0.0585 \mp 0.00048 \mathrm{eV}(\mathrm{NH})$ and $\sum m_{v}>$

$0.0986 \mp 0.00085 \mathrm{eV}(\mathrm{IH})$. Thus, our results are consistent of these experimental measurements.

\section{Conclusion and Discussion}

We propose the spin operator which vibrates harmonically in spin space and consider that this vibration generates the mass of neutrino. Along this consideration, we obtain the neutrino flavor states masses of which total mass is consistent to that of experimental measurement.

To explain the smallness of neutrino masses, widely accepted model is the seesaw mechanism as mentioned in introduction, however, the mass scale of the neutrino masses is unknown.

Recently, the possibility that they could be light enough to be produced and tested in laboratory experiments has been extensively discussed in the literature. One of this type of arguments is based on the displaced vertices [35-42]. Under this scenario, Hernandez et al. [43] explain the constraint to distinguish whether two heavy neutrino are pseudo-Dirac or 
Majorana neutrino based on the minimal type I seesaw model in normal hierarchy. In this case, if heavy neutrino are pseud-Dirac, the light neutrino mass splitting comes from higher order gauge interactions [44], and if heavy neutrino are Majorana neutrino, these two heavy neutrino causes the light neutrino mass splitting. For either cases, however, this mechanism may not explain the actual neutrino masses as shown as follows.

Because two light neutrino become massive in this case, one can obtain the light neutrino masses using the squared mass splitting $\Delta m_{21}^{2},\left|m_{31}^{2}\right|$ vales (see Table 2) as:

$$
\begin{gathered}
m_{1} c^{2}=0(\mathrm{eV}), m_{2} c^{2}=0.865 \times 10^{-2}(\mathrm{eV}), m_{3} c^{2} \\
=4.98 \times 10^{-2}(\mathrm{eV})
\end{gathered}
$$

Thus, the total mass becomes $\sum m_{l}=5.845 \times 10^{-2}(\mathrm{eV})$, which is smaller than the lower bound of the sum of neutrino masses for NH case. In the inverted hierarchy case, one can see the equivalent expression in Ref. [38, 44], if we consider the smallest case,

$$
\begin{gathered}
m_{3} c^{2}=0(\mathrm{eV}), m_{1} c^{2}=4.935 \times 10^{-2}(\mathrm{eV}), m_{2} c^{2} \\
=5.010 \times 10^{-2}(\mathrm{eV})
\end{gathered}
$$

Thus, the total mass becomes $\sum m_{l}=0.09945(\mathrm{eV})$, which is equal to largest lower bound of the sum of neutrino masses for IH case.

Thus, two heavy neutrino may not sufficient to explain the actual neutrino masses. Therefore, it is challenging target to explain the actual neutrino masses under seesaw model. On the other hand, in our estimation, we use the anomalous magnetic moment of leptons, which is described as the result of vibration of spin operator via the infinitesimal oscillation in azimuthal angle $\phi$ space, to obtain flavor state neutrino masses. Thus, electron neutrino and mu-neutrino masses are systemically estimated in section 4 . To estimate the remaining tau neutrino mass, we only need to find the actual anomalous magnetic moment of tauon. We know that the anomalous magnetic moment of leptons are calculated very precisely by quantum electrodynamics (QED). However, we consider that our description also gives some insight for understanding spin.

Although we obtain the consistent results, our argument is based on classical physics except the derivation of spin operator vibration. It is necessary to describe these phenomena fully by quantum theory.

\section{Appendix}

Here, we show that Eq. (45) is physical.

We can rewrite Eq. (40) as

$$
M_{v e} c^{2}=\frac{3}{8} \frac{\hbar c^{2}}{(\lambda \overline{\bar{\omega}} \prime)\left(\frac{1}{\lambda} a_{0}^{v e \prime}\right)}
$$

In other word, we change $\overline{\bar{\omega}}$ to $\lambda \overline{\bar{\omega}}^{\prime}$ and $a_{0}^{v e}$ to $\frac{1}{\lambda} a_{0}^{v e \prime}$ simultaneously.

Then Eq. (43) becomes as

$$
\frac{1}{2} M_{v e} c^{2}=\mu_{0}\left(\frac{2}{3} e\right)^{2} \frac{3}{4 \pi} \frac{(\lambda \overline{\bar{\omega} \prime})^{2}}{\frac{1}{\lambda} a_{0}^{v e \prime}}=\mu_{0}\left(\frac{2}{3} e\right)^{2} \frac{3}{4 \pi} \frac{\overline{\bar{\omega}} \prime^{2}}{a_{0}^{v e}} \lambda^{3}
$$

From Eq. (A1) and Eq. (A2), we can obtain

$$
\overline{\bar{\omega}}^{\prime}=\left(\frac{1}{2}\right)^{\frac{1}{3}}\left(\frac{9}{32 \alpha}\right)^{\frac{1}{3}} \frac{c}{\lambda}
$$

Then, taking $\lambda=\left(\frac{1}{2}\right)^{\frac{1}{3}}\left(\frac{9}{32 \alpha}\right)^{\frac{1}{3}}$, we obtain $\overline{\bar{\omega}}^{\prime}=\mathrm{c}$, which is physical.

In this case, the radius of electron neutrino $a_{0}^{v e}$ changes to $a_{0}^{\nu e^{\prime}}=\lambda 2 \sqrt{k} \frac{3 \hbar c}{\left(M_{e} c^{2}\right)^{\frac{3}{2}}} \sqrt{\frac{5}{4}}$.

Therefore, under these change, the value of $M_{v e} c^{2}$ is same as Eq. (45). Thus, Eq. (45) is physical.

\section{References}

[1] Fukuda Y et al. Phys. Rev. Lett. 81, 1562-1567 (1998).

[2] Ahmad Q. R. et al. Phys. Rev. Lett. 89, 011301 (2002).

[3] Araki T. et al. Phys. Rev. Lett. 94, 081801 (2005).

[4] Adamson P. et al. Phys. Rev. Lett. 101, 131802 (2008).

[5] Ahn J. K. et al. Phys. Rev. Lett. 108, 191802 (2012).

[6] Abe K. et al. Phys. Rev. Lett. 112, 061802 (2014).

[7] Loureiro A. et al. Phys. Rev. Lett. 123, 081301 (2019).

[8] Barabash A. S. Int. J. Mod. Phys. A 33, 1843001 (2018).

[9] Dolinski M. J., Poon A. W., and Rodejohann W. Ann. Rev. Nuclear and particle Science 69, (2019).

[10] Otten E. W. and Weinheimer C. Rep. Prog. Phys. 71, 086201 (2008).

[11] Drexlin G., Hannen V., Mertens S., and Weinheimer C. Adv. High Energy Phys. 2013 (2013).

[12] Gastaldo et al. Eur. Phys. J. ST. 226, 1623 (2017).

[13] Nicciot A. Adv. High Energy Phys. 2016, 9153024 (2016).

[14] Aker M et al., arXiv: 1909.06048vl 13 Sep 2019.

[15] Minkovski P. Phys. Lett. B 67, 421 (1977).

[16] Yanagida T. Conf. Proc. C79023131, 95 (1979).

[17] Gell-mann M., Ramond P., and Stansky R., Conf. Proc. C790927, 315 (1979).

[18] Mohaparta R. N. and Senjanvic G., Phys. Rev. Lett. 44, 912 (1981).

[19] Glashow S. L., 1980 NATO adv. Study Inst. Ser. B Phys. 59, 687 (1980).

[20] Morisi S. and Peinad E., Phys. Rev. D 80, 113011 (2009).

[21] Morisi S, Peinad E. Shimizu Y. and Valle J. W., Phys. Rev. D 84, 036003 (2011).

[22] Suura H., Phys. Re. D 17, 469 (1978). 
[23] Suura H. Phys. Rev. D 20, 1429 (1979).

[24] Kurai T. Results in Phys, 10. 865-881 (2018).

[25] Moriguch S, Udagawa K. and Hitotsumatsu S., Formula of Mathematics III; Special Functions, Iwanami (1975).

[26] Weinberg S., Quantum Theory of Fields (III), Cambridge Univ. Press (2000).

[27] Tomonaga S., The story of spin, Univ. of Chicago Press (1998).

[28] Hanneke D., Fogwell Hoogerheide S. and Gabrielse G., Phys. Rev. A 83 (5), 052122 (2011).

[29] Palrignani C. and Agahe K., Chinese Phys. C IOP Publishing 40 (10), 100001 (2016).

[30] Gonzalez M. C., Maltoni M. and Schwez T., J. High Energy Phys. 52 (2014).

[31] Hannestad S. and Schwez T., J. Cosmol. Astropart. Phys. 11,035 (2016).

[32] Choudhury S. R. and Choubey S., J. Cosmol. Astropart. Phys. 2018, 017 (2018).

[33] Long A. J., Raveri M., Hu W. and Dodelson S., Phys. Rev. D 97, 043510 (2018).

[34] Gariazzo S., Archidiacono M., de Salas P. F., Mena O., Ternes C. A., and Tortola M., J. Cosmol. Astropart. Phys. 2018, 011 (2018).
[35] Helo J. C., Hirsch M., and Kovalenko S., Phys. Rev. D 89, 073005 (20149 [Erratum: Phys. Rev. D 93, no. 9, 099902 (2016)].

[36] Blondel A., Graverini E., Serra N., et al. (FCC-ee study Team), Nucl. Part. Phys. Proc. 273-275, 1883 (2016).

[37] Cui Y., and Shuve B., JHEP 02, 049 (2015).

[38] Gago A. M., Hernandez P., Jones-Perez J., et al. Eur Phys. J. C $75,10,475,(2015)$.

[39] Duarte L., Peressetti J., and Sampayo O. A., J. Phys. G 45, 2, 025001 (2018).

[40] Antush S., Cazzato E., and Fischer O., JHEP 12, 007, (2016).

[41] Caputo A., Hernendez P., Lopez-Pavon J., et al. JHEP 06, 112 (2017).

[42] Antush S., Cazzato E., and Fischer O., Phys. Lett. B 774, 114 (2017).

[43] Hernandez P., Jones-Perez J., and Suanrez-Navarro O., Eur. Phys. J. C 79, 3, 220 (2019).

[44] Lindner M., Ohlsson T., and Seidl G., arXiv: hep-ph/0109264v2 17 Nov 2001.

[45] Donini A., Hernandez P., and Lopez-Pavon J., JHEP 07, 161 (2012). 